\title{
Emerging infectious diseases associated with bat viruses
}

\author{
SHI ZhengLi \\ Center for Emerging Infectious Diseases, State Key Laboratory of Virology, Wuhan Institute of Virology, Chinese Academy of Sciences, \\ Wuhan 430071, China
}

Received May 27, 2013; accepted June 14, 2013

\begin{abstract}
Bats play important roles as pollen disseminators and pest predators. However, recent interest has focused on their role as natural reservoirs of pathogens associated with emerging infectious diseases. Prior to the outbreak of severe acute respiratory syndrome (SARS), about 60 bat virus species had been reported. The number of identified bat viruses has dramatically increased since the initial SARS outbreak, and most are putative novel virus species or genotypes. Serious infectious diseases caused by previously identified bat viruses continue to emerge throughout in Asia, Australia, Africa and America. Intriguingly, bats infected by these different viruses seldom display clinical symptoms of illness. The pathogenesis and potential threat of bat-borne viruses to public health remains largely unknown. This review provides a brief overview of bat viruses associated with emerging human infectious diseases.
\end{abstract}

emerging infectious disease, bat virus, natural reservoirs

Citation: $\quad$ Shi Z L. Emerging infectious diseases associated with bat viruses. Sci China Life Sci, 2013, 56: 678-682, doi: 10.1007/s11427-013-4517-x

\section{Bat coronaviruses}

Members of the family Coronaviridae, within the order Nidovirales, are enveloped, positive-stranded RNA viruses. Several genera have been created for this order of viruses, including Alphacoronaviruses and Betacoronaviruses, whose members infect mammals; and Gammacoronaviruses and Deltaoronaviruses, which affect both avian and mammalian species [1,2]. Coronavirus infection usually causes a mild upper respiratory syndrome in humans, however, pathogens that are transmitted zoonotically can be highly pathogenic. This was seen with severe acute respiratory syndrome (SARS) in 2002-2003, which was caused by a novel coronavirus (SARS-CoV) [3,4]. It was demonstrated that SARS-CoV was transmitted to humans from the market civet [5-7]. However, studies of farmed and wild civets did not support the theory that civets were the natural reservoirs

email: zlshi@wh.iov.cn of SARS-CoV [7-9]. In 2005, two independent teams reported the discovery of SARS-like CoVs (SL-CoVs) in bats and suggested that bats are natural reservoirs of SARS-CoV $[10,11]$. Since then, more genetically diverse SL-CoVs have been found in China, Europe and Africa, suggesting a wide geographical distribution and an ancient origin for these viruses [12-15]. The majority of bat SL-CoVs were found in rhinolopus bats, particularly Rhinolophus sinicus, and display $87 \%-92 \%$ nucleic acid identity and $93 \%-100 \%$ amino acid similarity with SARS-CoV [10,11,14-16]. However, those isolates found in Europe and Africa are distantly related to SARS-CoV at a genomic level and possibly represent a new coronavirus species $[12,13,16]$. Unfortunately none of the above SL-CoVs use the same receptor as SARS-CoV, angiotensin converting enzyme II, to facilitate virus entry into a cell. This suggests that these SL-CoVs are not direct progenitors of SARS-CoV [17]. Phylogenetic analyses support the recombinant origin of a bat SL-CoV coronavirus, and that $R$. sinicus may carry the 
director progenitor of SARS-CoV [18]. Therefore, wideranging surveillance of SL-CoVs in bats is needed to determine the origin of these lethal viruses.

In 2012, a disease presenting with acute pneumonia and subsequent renal failure, symptoms similar to those for SARS, emerged in Saudi Arabia. A novel human coronavirus (MERS-CoV, Middle East respiratory syndrome human coronavirus) was suspected to be responsible for this fatal disease. Globally, 64 human cases were confirmed resulting in 38 deaths by June 17, 2013 [19,20]. Phylogenetic analysis showed that MERS-CoV belongs to lineage $\mathrm{C}$ of the Betacoronavirus genus. It was most similar to the bat coronaviruses HKU4 and HKU5, which infect the lesser bamboo bat (Tylonycteris pachypus) and pipistrelle bat (Pipistrellus pipistrellus), respectively [21,22]. MERS-CoV exhibits $50 \%$ nucleotide identity with the entire genomic sequences of HKU4 and HKU5, and 82\% nucleotide identity with the RNA dependent RNA polymerase (RdRp) gene. A recent study confirmed that MERS-CoV uses dipeptidyl peptidase 4 (DPPIV), also known as CD26, as a functional receptor. It has also been shown that this molecule is evolutionarily conserved among mammals, and MERS-CoV can infect a broad range of mammalian cells (humans, pigs, monkeys and bats), suggesting ease of cross-host transmission [23,24]. Whether MERS-CoV was directly transmitted from bats to humans requires further research to confirm.

Human coronavirus 229E (HCoV-229E) and NL63 (HCoV-NL63) belong to the genus Alphacoronavirus. These were discovered in 1970 and 2004, respectively, and lead to flu-like symptoms and bronchiolitis [25,26]. In 2009, a bat CoV (Hipposideros/GhanaKwam/19/2008) was discovered in the leaf-nosed bat (Hipposideros caffer ruber) in Ghana and was predicted to share a most recent common ancestor (MRCA) from just over 200 hundred years ago with HCoV-229E. A fragment of RdRp in bat CoV Hipposideros/GhanaKwam/19/2008 shared 92\% nucleic acid identity with $\mathrm{HCoV}-229 \mathrm{E}$ [27]. Most recently, a bat $\mathrm{CoV}$ (Bat CoV Perimyotis subflavus strain ARCoV.2) was detected in the North American tricolored bat (Perimyotis subflavus) and was predicted to share common ancestry with HCoV-NL63; the MRCA between these viruses was predicted to have occurred approximately 563-822 years ago [28]. Phylogenetic analyses of all known CoVs in mammals suggested that bats are ideal hosts for both $A l$ phacoronaviruses and Betacoronaviruses [1].

\section{Bat lyssaviruses}

Lyssaviruses are negative-sense RNA, bullet-shaped viruses within the Rhabdoviridae family, of the order Mononegavirale. These viruses cause acute progressive encephalitis (rabies) that is inevitably fatal once clinical signs develop in mammals. There are 12 lyssavirus genotypes: rabies virus (RABV); Aravan virus (ARAV); Australian bat lyssavirus
(ABLV); Duvenhage virus (DUVV); European bat lyssavirus type 1 (EBLV-1); European bat lyssavirus type 2 (EBLV-2); Irkut virus (IRKV); Khujand virus (KHUV); Lagos bat virus (LBV); Mokola virus (MOKV); Shimoni bat virus (SHIBV); and West caucasian bat virus (WCBV) [29]. The link between vampire bat bites and rabies was established at the beginning of the 20th century. Since then, a number of confirmed or suspicious rabies cases have been associated with bats in the USA, Canada, Australia, Latin America, Western Europe and China [29-31]. All lyssaviruses, except Mokola virus, involve bat reservoirs that include fruit bats (Rousettus aegyptiacus and Eidolon helvum) and microbats (Miniopterus schreibersiias, Tadarida brasiliensis, Hipposideros commersoni and Myotis spp.). However, the number of human deaths due to bat rabies remains small compared with those from dog bites in countries with poor sanitary conditions.

\section{Bat paramyxoviruses}

Over the past decade a number of zoonotic viruses have emerged from flying foxes, and these have been shown to cause serious disease outbreaks in humans and livestock. Hendra virus (HeV) was identified in 1994 as the causative agent of an acute respiratory disease in horses in Brisbane (Australia), and was associated with a single fatality in humans [32]. $\mathrm{HeV}$ is naturally hosted by fruit bats (Pteropus species), and poses a serious threat to livestock in Australia, with sporadic lethal transmissions to humans almost every year [33]. In 1998 in Malaysia, the closely related Nipah virus $(\mathrm{NiV})$ was found to infect pigs and humans, inducing encephalitis with $40 \%$ fatality [34]. Almost every year since, outbreaks of NiV cause severe encephalitis in Bangladesh and India with fatality rate approaching 75\% [35]. Multiple rounds of person-to-person $\mathrm{NiV}$ transmission have been observed [35,36], increasing the likelihood of NiV infection in humans. In addition to acute infection, these viruses also cause asymptomatic infections that may lead to late-onset or relapsing encephalitis years after the initial infection [37]. Although $\mathrm{NiV}$ appears to be most closely related to the morbilliviruses, a few properties of $\mathrm{NiV}$ and $\mathrm{HeV}$, including their large genome size, have led to their classification within the Henipavirus genus of the Paramyxoviridae family [38]. Because of their ability to infect humans, high pathogenicity, wide host range and potent interspecies transmission, $\mathrm{HeV}$ and $\mathrm{NiV}$ are classified as biosecurity level 4 pathogens.

Apart from viral species that cause fatal diseases in animals and humans in Australia, Malaysia, Bangladesh and India, new distinct viral clades closely related to $\mathrm{HeV}$ and $\mathrm{NiV}$ have been identified in South Asian and African countries [39-41]. Although many of these pathogens are capable of inducing severe systemic illnesses in diverse terrestrial mammalian hosts, they are comparatively innocuous in 
bats $[42,43]$. Under experimental conditions, it has been shown that the infection of bats with a range of viruses rarely results in disease. Within tissues, low levels of virus are detected, viral shedding is often at the limit of detection, and inconsistent or transient seroconversion is observed $[43,44]$.

\section{Bat filoviruses}

Filoviruses are associated with acute fatal hemorrhagic diseases of humans and/or nonhuman primates. The Filoviridae family comprises two genera: Marburgviruses (MARV) and Ebolaviruses (EBOV) [45]. MARV was first identified in 1967 during hemorrhagic epidemics in Germany and the former Yugoslavia following the importation of infected monkeys from Uganda [46]. The five known MARV lineages have been classified as being made up of two viruses, Ravn virus and MARV [47]. EBOV was first identified in the western equatorial province of Sudan in 1976. Five distinct species of EBOV have been identified: Ivory Coast ebolavirus (CIEBOV); Sudan ebolavirus (SEBOV); Zaire ebolavirus (ZEBOV); Reston ebolavirus (REBOV); and Bundibugyo ebolavirus (proposed) [45]. The Bundibugyo, Sudan and Zaire species have been associated with large outbreaks of Ebola haemorrhagic fever (EHF). These viruses are among the most virulent pathogens known to infect humans. EBOV or MARV outbreaks regularly occur in African countries with a high fatality rate (25\%-90\%) (http://www.who.int/csr/disease).

Bats have been implicated as the reservoirs for MARV and EBOV. Serological and genetic data has shown that these viruses have been detected in the common Egyptian fruit bat Rousettus aegyptiacus, the tree-roosting fruit bat Eidolon helvum, and other African bat species [47-51]. Almost all primary infections related to MARV have been linked to humans entering caves inhabited by bats [47]. MARV and EBOV sequences detected in bats are closely related to previously isolated MARV or EBOV sequences and those detected in humans [47,52].

\section{Bat reoviruses}

The Reoviridae is a family of viruses that can infect a wide range of hosts, from plants to mammals. The genus Orthoreovirus is within the Spinareovirinae and contains five virus species: Pteropine orthoreovirus; Avian orthoreovirus; Reptilian orthoreovirus; Baboon orthoreovirus; and the type species Mammalian orthoreovirus (MRV). Orthoreovirus infection often occurs in animals and humans, but most cases are mild. The first reported bat reovirus, Pteropine orthoreovirus, was isolated from the blood of a fruit bat or "flying fox" (Pteropus poliocephalus) in New South Wales (Australia) [53]. Thirty years later, a virus similar to Ptero- pine orthoreovirus, which was named Pulau virus, was isolated and identified during the search for natural reservoirs of NiV [54]. Similar viruses were also isolated from the Chinese fruit bat Rousettus leschenaultia [55]. Bat-borne orthoreoviruses received increased attention after several orthroviruses (Melaka virus and Kampar virus) were isolated from patients with acute respiratory syndrome in Malaysia in 2007 [56,57], and from three individuals in Hong Kong between 2007 and 2010 [58]. The reoviruses isolated from patients were closely related to those discovered in bats. Epidemiological studies have also indicated that these reoviruses are probably of bat origin. In addition, mammalian orthoreovirus have been isolated from European microbats $[59,60]$, suggesting a high probability of interspecies transmission between animals.

\section{Other novel bat viruses}

A large number of novel bat viruses have been discovered worldwide and classified into various families, including the Adenoviridae [61,62]; Astroviridae [63,64]; Coronaviridae [65-71]; Circoviridae [72]; Filoviridae [73]; Flaviviridae [74,75]; Hepadnaviridae [76]; Hepeviridae [75,77]; Herpesviridae [78]; Orthomyxoviridae [79,80]; Parvoviridae [81]; Picornaviridae [82]; Papillomaviridae [83]; Paramyxoviridae [41]; and Poxviridae [84]. Most of these novel viruses are distantly related to known animal or human viruses. There exists huge genetic diversity among these novel bat viruses, regardless of whether the genome was RNA or DNA. However, because virus isolation has rarely been successful for most bat viruses, the pathogenesis of these viruses remains unclear. None of the aforementioned viruses has been confirmed to be prevalent in human populations. Although the direct transmission of viruses from wild animals to humans is rare, close attention needs to be paid to the potential threat these novel viruses pose to the public.

It is of interest to note that bats, whether naturally or experimentally infected with viruses, seldom display clinical symptoms. This has been associated with the unique characteristics of the bat immune system. Recently, two bat genomes (Pteropus alecto and Myotis davidii) have been fully sequenced [85], and an unexpected concentration of positively selected genes were discovered at the DNA damage checkpoint and nuclear factor kB pathway. The DNA damage response plays an important role in host defense and is a known target for virus interaction [86]. This raises the possibility that changes in DNA damage response mechanisms during selection could have influenced the bat immune system. Additionally, several important genes responsible for responding to microbe infections in mammals are lost in both $P$. alecto and $M$. davidii, indicating that immune functions in bats likely differ compared with that in other mammals. 
1 Woo P C Y, Lau S K P, Lam C S F, et al. Discovery of seven novel mammalian and avian coronaviruses in the genus Deltacoronavirus supports bat coronaviruses as the gene source of Alphacoronavirus and Betacoronavirus and avian coronaviruses as the gene source of Gammacoronavirus and Deltacoronavirus. J Virol, 2012, 86: 39954008

2 de Groot R, Baker S, Baric R, et al. Family Coronaviridae. In: Virus Taxonomy: Ninth Report of the International Committee on Taxonomy of Viruses. San Diego, CA: Academic Press, 2012. 806-828

3 Ksiazek T G, Erdman D, Goldsmith C S, et al. A novel coronavirus associated with severe acute respiratory syndrome. N Engl J Med, 2003, 348: 1953-1966

4 Drosten C, Gunther S, Preiser W, et al. Identification of a novel coronavirus in patients with severe acute respiratory syndrome. $\mathrm{N}$ Engl J Med, 2003, 348: 1967-1976

5 Guan Y, Zheng B J, He Y Q, et al. Isolation and characterization of viruses related to the SARS coronavirus from animals in southern China. Science, 2003, 302: 276-278

6 Wang M, Yan M, Xu H, et al. SARS-CoV infection in a restaurant from palm civet. Emerg Infect Dis, 2005, 11: 1860-1865

7 Tu C, Crameri G, Kong X, et al. Antibodies to SARS coronavirus in civets. Emerg Infect Dis, 2004, 10: 2244-2248

8 Kan B, Wang M, Jing H, et al. Molecular evolution analysis and geographic investigation of severe acute respiratory syndrome coronavirus-like virus in palm civets at an animal market and on farms. J Virol, 2005, 79: 11892-11900

9 Poon L L M, Chu D K W, Chan K H, et al. Identification of a novel coronavirus in bats. J Virol, 2005, 79: 2001-2009

$10 \mathrm{Li} \mathrm{W} \mathrm{D,} \mathrm{Shi} \mathrm{Z} \mathrm{L,} \mathrm{Yu} \mathrm{M,} \mathrm{et} \mathrm{al.} \mathrm{Bats} \mathrm{are} \mathrm{natural} \mathrm{reservoirs} \mathrm{of}$ SARS-like coronaviruses. Science, 2005, 310: 676-679

11 Lau S K P, Woo P C Y, Li K S M, et al. Severe acute respiratory syndrome coronavirus-like virus in Chinese horseshoe bats. Proc Natl Acad Sci USA, 2005, 102: 14040-14045

12 Drexler J F, Gloza-Rausch F, Glende J, et al. Genomic characterization of severe acute respiratory syndrome-related coronavirus in European bats and classification of coronaviruses based on partial RNA-dependent RNA polymerase gene sequences. J Virol, 2010, 84: 11336-11349

13 Tong S, Conrardy C, Ruone S, et al. Detection of novel SARS-like and other coronaviruses in bats from Kenya. Emerg Infect Dis, 2009, 15: 482-485

14 Yuan J, Hon C C, Li Y, et al. Intraspecies diversity of SARS-like coronaviruses in Rhinolophus sinicus and its implications for the origin of SARS coronaviruses in humans. J Gen Virol, 2010, 91: 1058-1062

15 Ren W, Li W D, Yu M, et al. Full-length genome sequences of two SARS-like coronaviruses in horseshoe bats and genetic variation analysis. J Gen Virol, 2006, 87: 3355-3359

16 Quan P L, Firth C, Street C, et al. Identification of a severe acute respiratory syndrome coronavirus-like virus in a leaf-nosed bat in Nigeria. MBio, 2010, e00208-10

17 Ren W, Qu X X, Li W D, et al. Difference in receptor usage between severe acute respiratory syndrome (SARS) coronavirus and SARSlike coronavirus of bat origin. J Virol, 2008, 82: 1899-1907

18 Hon C C, Lam T Y, Shi Z L, et al. Evidence of the recombinant origin of a bat severe acute respiratory syndrome (SARS)-like coronavirus and its implications on the direct ancestor of SARS coronavirus. J Virol, 2008, 82: 1819-1826

19 WHO. Coronavirus infections. http://www.who.int/csr/disease/coronavirus_infections/en/

20 Zaki A M, van Boheemen S, Bestebroer T M, et al. Isolation of a novel coronavirus from a man with pneumonia in Saudi Arabia. N Engl J Med, 2012, 367: 1814-1820

21 Lau S K, Li K S, Tsang A K, et al. Genetic characterization of Betacoronavirus lineage $\mathrm{C}$ viruses in bats revealed marked sequence divergence in the spike protein of Pipistrellus bat coronavirus HKU5 in Japanese pipistrelle: implications on the origin of the novel Middle East Respiratory Syndrome Coronavirus. J Virol, 2013, doi: 10.1128/JVI.01055-13
22 van Boheemen S, de Graaf M, Lauber C, et al. Genomic characterization of a newly discovered coronavirus associated with acute respiratory distress syndrome in humans. MBio, 2012, 3: e00473-12

23 Raj V S, Mou H, Smits S L, et al. Dipeptidyl peptidase 4 is a functional receptor for the emerging human coronavirus-EMC. Nature, 2013, 495: 251-254

24 Lu GW, Liu D. SARS-like virus in the middle east: A truly bat-related coronavirus causing human diseases. Protein Cell, 2012, 3: 803-805

25 van der Hoek L, Pyrc K, Jebbink M F, et al. Identification of a new human coronavirus. Nat Med, 2004, 10: 368-373

26 Cavallar J J, Monto A S. Community-wide outbreak of infection with a 229E-like coronavirus in Tecumseh, Michigan. J Infect Dis, 1970, 122: $272-279$

27 Pfefferle S, Oppong S, Drexler J F, et al. Distant relatives of severe acute respiratory syndrome coronavirus and close relatives of human coronavirus 229E in bats, Ghana. Emerg Infect Dis, 2009, 15: 1377-1384

28 Huynh J, Li S, Yount B, et al. Evidence supporting a zoonotic origin of human coronavirus strain NL63. J Virol, 2012, 86: 12816-12825

29 Johnson N, Vos A, Freuling C, et al. Human rabies due to lyssavirus infection of bat origin. Vet Microbiol, 2010, 142: 151-159

30 Rupprecht C E, Turmelle A, Kuzmin I V. A perspective on lyssavirus emergence and perpetuation. Cur Opin Virol, 2011, 1: 662-670

31 Tang X, Luo M, Zhang S, et al. Pivotal role of dogs in rabies transmission, China. Emerg Infect Dis, 2005, 11: 1970-1972

32 Murray K, Selleck P, Hooper P, et al. A morbillivirus that caused fatal disease in horses and humans. Science, 1995, 268: 94-97

33 Field H, Crameri G, Kung N Y, et al. Ecological aspects of Hendra virus. Cur Top Microbiol Immunol, 2012, 359: 11-23

34 Chua K B, Bellini W J, Rota P A, et al. Nipah virus: a recently emergent deadly paramyxovirus. Science, 2000, 288: 1432-1435

35 Lo M K, Rota P A. The emergence of Nipah virus, a highly pathogenic paramyxovirus. J Clin Virol, 2008, 43: 396-400

36 Luby P S, Hossain M J, Gurley E S, et al. Recurrent zoonotic transmission of Nipah virus into humans, Bangladesh, 2001-2007. Emerg Infect Dis, 2009, 15: 1229-1233

37 Tan C T, Goh K J, Wong K T, et al. Relapsed and late-onset Nipah encephalitis. Ann Neurol, 2002, 51: 703-708

38 Wang L F, Yu M, Hansson E, et al. The exceptionally large genome of Hendra virus: support for creation of a new genus within the family Paramyxoviridae. J Virol, 2000, 74: 9972-9979

39 Reynes J M, Counor D, Ong S, et al. Nipah virus in lyle's flying foxes, Cambodia. Emerg Infect Dis, 2005, 11: 1042-1047

40 Wacharapluesadee S, Lumlertdacha B, Boongird K, et al. Bat Nipah virus, Thailand. Emerg Infect Dis, 2005, 11: 1949-1951

41 Drexler J F, Corman V M, Muller M A, et al. Bats host major mammalian paramyxoviruses. Nat Commun, 2012, 3: 796

42 Shi Z. Bat and virus. Protein Cell, 2010, 1: 109-114

43 Calisher C H, Childs J E, Field H E, et al. Bats: important reservoir hosts of emerging viruses. Clin Microbiol Rev, 2006, 19: 531-545

44 Wong S, Lau S, Woo P, et al. Bats as a continuing source of emerging infections in humans. Rev Med Virol, 2007, 17: 67-91

45 Barrette R W, Xu L, Rowland J M, et al. Current perspectives on the phylogeny of Filoviridae. Infect Genet Evol, 2011, 11: 1514-1519

46 Slenczka W, Klenk H D. Forty years of Marburg virus. J Infect Dis, 2007, 196(Suppl 2): S131-S135

47 Towner J S, Amman B R, Sealy T K, et al. Isolation of genetically diverse Marburg viruses from Egyptian fruit bats. PLoS Pathog, 2009, 5: e1000536

48 Leroy E M, Kumulungui B, Pourrut X, et al. Fruit bats as reservoirs of Ebola virus. Nature, 2005, 438: 575-576

49 Towner J S, Pourrut X, Albarino C G, et al. Marburg virus infection detected in a common African bat. PLoS ONE, 2007, 2: e764

50 Hayman D T, Emmerich P, Yu M, et al. Long-term survival of an urban fruit bat seropositive for Ebola and Lagos bat viruses. PLoS ONE, 2010, 5: e11978 
51 Pourrut X, Souris M, Towner J S, et al. Large serological survey showing cocirculation of ebola and marburg viruses in gabonese bat populations, and a high seroprevalence of both viruses in Rousettus aegyptiacus. BMC Infect Dis, 2009, 9: 159

52 Biek R, Walsh P D, Leroy E M, et al. Recent common ancestry of Ebola zaire virus found in a bat reservoir. PLoS Pathog, 2006, 2: e90

53 Gard G P, Marshall I D. Nelson bay virus. A novel reovirus. Arch Gesamte Virusforsch, 1973, 43: 34-42

54 Pritchard L I, Chua K B, Cummins D, et al. Pulau virus: a new member of the Nelson bay orthoreovirus species isolated from fruit bats in Malaysia. Arch Virol, 2006, 151: 229-239

$55 \mathrm{Du} \mathrm{L}, \mathrm{Lu} \mathrm{Z}$, Fan Y, et al. Xi river virus, a new bat reovirus isolated in southern China. Arch Virol, 2010, 155: 1295-1299

56 Chua K B, Voon K, Crameri G, et al. Identification and characterization of a new orthoreovirus from patients with acute respiratory infections. PLoS ONE, 2008, 3: e3803

57 Chua K B, Crameri G, Hyatt A, et al. A previously unknown reovirus of bat origin is associated with an acute respiratory disease in humans. Proc Nat Acad Sci USA, 2007, 104: 11424-11429

58 Wong A H, Cheng P K, Lai M Y, et al. Virulence potential of fusogenic orthoreoviruses. Emerg Infect Dis, 2012, 18: 944-948

59 Kohl C, Lesnik R, Brinkmann A, et al. Isolation and characterization of three mammalian orthoreoviruses from European bats. PLoS ONE, 2012, 7: e43106

60 Lelli D, Moreno A, Lavazza A, et al. Identification of mammalian orthoreovirus type 3 in Italian bats. Zoo Pub Health, 2012, 60: 84-92

61 Li Y, Ge X, Zhang H, et al. Host range, prevalence, and genetic diversity of adenoviruses in bats. J Virol, 2010, 84: 3889-3897

62 Maeda K, Hondo E, Terakawa J, et al. Isolation of novel adenovirus from fruit bat (Pteropus dasymallus yayeyamae). Emerg Infect Dis, 2008, 14: 347-349

63 Xiao J, Li J, Hu G, et al. Isolation and phylogenetic characterization of bat astroviruses in southern China. Arch Virol, 2011, 156: 1415-1423

64 Zhu H C, Chu D K, Liu W, et al. Detection of diverse astroviruses from bats in china. J Gen Virol, 2009, 90: 883-887

65 Tsuda S, Watanabe S, Masangkay J S, et al. Genomic and serological detection of bat coronavirus from bats in the Philippines. Arch Virol, 2012, 157: 2349-2355

66 Lau S K P, Li K S M, Tsang A K L, et al. Recent transmission of a novel alphacoronavirus, bat coronavirus HKU10, from leschenault's rousettes to pomona leaf-nosed bats: first evidence of interspecies transmission of coronavirus between bats of different suborders. J Virol, 2012, 86: 11906-11918

67 August T A, Mathews F, Nunn M A. Alphacoronavirus detected in bats in the United Kingdom. Vector-Borne Zoonot, 2012, 12: 530-533

68 Tao Y, Tang K, Shi M, et al. Genomic characterization of seven distinct bat coronaviruses in Kenya. Virus Res, 2012, 167: 67-73

69 Lau S K P, Poon R W S, Wong B H L, et al. Coexistence of different genotypes in the same bat and serological characterization of rousettus bat coronavirus HKU9 belonging to a novel betacoronavirus subgroup. J Virol, 2010, 84: 11385-11394

70 Watanabe S, Masangkay J S, Nagata N, et al. Bat coronaviruses and experimental infection of bats, the Philippines. Emerg Infect Dis, 2010, 16: 1217-1223

71 Donaldson E F, Haskew A N, Gates J E, et al. Metagenomic analysis of the viromes of three north American bat species: viral diversity among different bat species that share a common habitat. J Virol, 2010, 84: 13004-13018

72 Ge X, Li J, Peng C, et al. Genetic diversity of novel circular ssDNA viruses in bats in China. J Gen Virol, 2012, 92: 2646-2653

73 Negredo A, Palacios G, Vazquez-Moron S, et al. Discovery of an Ebolavirus-like filovirus in Europe. PLoS Pathog, 2011, 7: e1002304

$74 \mathrm{Wu}$ Z, Ren X, Yang L, et al. Virome analysis for identification of novel mammalian viruses in bat species from Chinese provinces. $\mathrm{J}$ Virol, 2012, 86: 10999-11012

75 Quan P L, Firth C, Conte J M, et al. Bats are a major natural reservoir for hepaciviruses and pegiviruses. Proc Natil Acad Sci USA, 2013, 110: 8194-8199

76 He B, Li Z, Yang F, et al. Virome profiling of bats from Myanmar by metagenomic analysis of tissue samples reveals more novel mammalian viruses. PLoS ONE, 2013, 8: e61950

77 Drexler J F, Seelen A, Corman V M, et al. Bats worldwide carry hepatitis $\mathrm{E}$ virus-related viruses that form a putative novel genus within the family Hepeviridae. J Virol, 2012, 86: 9134-9147

78 Zhang H, Todd S, Tachedjian M, et al. A novel bat herpesvirus encodes homologues of major histocompatibility complex classes I and II, c-type lectin, and a unique family of immune-related genes. J Virol, 2012, 86: 8014-8030

79 Tong S, Li Y, Rivailler P, et al. A distinct lineage of influenza a virus from bats. Proc Natil Acad Sci USA, 2012, 109: 4269-4274

80 Sun X, Shi Y, Lu X, et al. Bat-derived influenza hemagglutinin H17 does not bind canonical avian or human receptors and most likely uses a unique entry mechanism. Cell Rep, 2013, 3: 769-778

81 Ge X, Li Y, Yang X, et al. Metagenomic analysis of viruses from bat fecal samples reveals many novel viruses in insectivorous bats in China. J Virol, 2012, 86: 4620-4630

82 Lau S K, Woo P C, Lai K K, et al. Complete genome analysis of three novel picornaviruses from diverse bat species. J Virol, 2011, 85: 8819-8828

83 McKnight C A, Wise A G, Maes R K, et al. Papillomavirusassociated basosquamous carcinoma in an Egyptian fruit bat (Rousettus aegyptiacus). J Zoo Wildl Med, 2006, 37: 193-196

84 Li L, Victoria J G, Wang C, et al. Bat guano virome: predominance of dietary viruses from insects and plants plus novel mammalian viruses. J Virol, 2010, 84: 6955-6965

85 Zhang G, Cowled C, Shi Z, et al. Comparative analysis of bat genomes provides insight into the evolution of flight and immunity. Science, 2013, 339: 456-460

86 Turnell A S, Grand R J. DNA viruses and the cellular DNA-damage response. J Gen Virol, 2012, 93: 2076-2097

Open Access This article is distributed under the terms of the Creative Commons Attribution License which permits any use, distribution, and reproduction in any medium, provided the original author(s) and source are credited. 